\title{
SHARP CONTROL TIME FOR VISCOELASTIC BODIES
}

\author{
L. PANDOLFI
}

Communicated by Masahiro Yamamoto

\begin{abstract}
The evolution in time of a viscoelastic body is described by an equation with memory, which can be seen as a perturbation of the equations of elasticity. This observation is a useful tool in the study of control problems. In this paper, by using moment methods, we compare a viscoelastic system which fills a surface or a solid region (the string case has already been studied) with its elastic counterpart (which is a generalized telegrapher's equation) in order to prove exact controllability of the viscoelastic body as a consequence of the assumed controllability of the associated telegrapher's equation.
\end{abstract}

1. Introduction. We study a control problem for the following equation:

$$
\begin{aligned}
& w_{t t}=2 c w_{t}+\nabla \cdot(a(x) \nabla w)+q(x) w \\
& +\int_{0}^{t} M(t-s)\{\nabla \cdot(a(x) \nabla w(s))+q(x) w(s)\} \mathrm{d} s+F(x, t) .
\end{aligned}
$$

Here $w=w(x, t): \Omega \times[0, T] \mapsto \mathbb{R}$ where $T>0$ and $\Omega \subseteq \mathbb{R}^{d}, d \leq 3$.

Equation (1.1) has several interpretations. For every $d, w(x, t)$ represents the temperature of a thermodynamical system with memory which occupies the region $\Omega$, see [12]. In linear viscoelasticity and when $d=1$ or $d=2, w(x, t)$ represents the displacement of the point

2010 AMS Mathematics subject classification. Primary 45K05, 93B03, 93B05, $93 \mathrm{C} 22$.

Keywords and phrases. Controllability and observability, integral equations, linear systems, partial differential equations, heat equations with memory, viscoelasticity.

This paper fits into the research programme of the GNAMPA-INDAM and has been written in the framework of the "Groupement de Recherche en Contrôle des EDP entre la France et l'Italie (CONEDP-CNRS)".

Received by the editors on May 6, 2013, and in revised form on April 30, 2014. DOI: 10.1216/JIE-2015-27-1-103 
in position $x$ at time $t$ of a body which fills the region $\Omega$ (see [31]). If $d=3$, then a similar interpretation holds for quite special classes of displacements.

Equation (1.1) has to be supplemented with the initial condition

$$
w(\cdot, 0)=w_{0}, \quad w_{t}(\cdot, 0)=w_{1} .
$$

A control $f \in L_{\text {loc }}^{2}\left(0,+\infty ; L^{2}(\Gamma)\right)$ acts on the boundary of $\Omega$ :

$$
w(x, t)=f(x, t), \quad x \in \Gamma \subseteq \partial \Omega, \quad w(x, t)=0, \quad x \in \partial \Omega \backslash \Gamma .
$$

We stress the fact that the control $f$ is real valued.

Note that the arguments of $w=w(x, t)$ are not explicitly indicated unless needed for clarity. We shall write $w(x, t)$ or $w(t)$, or simply $w$. Furthermore, $w$ does depend on $f$ but this dependence is not indicated.

We refer to [14] for the following properties of (1.1) (see also [24, Appendix]). Let $f \in L^{2}\left(G_{T}\right)=L^{2}\left(0, T ; L^{2}(\Gamma)\right)$ and $w(\cdot, 0)=\xi \in$ $L^{2}(\Omega), w_{t}(\cdot, 0)=\eta \in H^{-1}(\Omega)$. Then, (1.1) admits a unique solution $w(\cdot, t) \in C\left(0, T ; L^{2}(\Omega)\right) \cap C^{1}\left(0, T ; H^{-1}(\Omega)\right)$, and the transformation

$$
(\xi, \eta, f) \longmapsto\left(w, w_{t}\right)
$$

is linear and continuous in the indicated spaces. So, the following definition of controllability is justified:

Definition 1.1. System (1.1) is controllable at time $T$ if, for every $w_{0}$, $\xi \in L^{2}(\Omega)$ and $w_{1}, \eta \in H^{-1}(\Omega)$, there exists $f \in L^{2}\left(0, T ; L^{2}(\Gamma)\right)$ such that

$$
w(\cdot, T)=\xi \in L^{2}(\Omega), \quad w_{t}(\cdot, T)=\eta \in H^{-1}(\Omega) .
$$

A control $f$ with this property is called a steering control (to the target $(\xi, \eta))$.

It is known that controllability of a linear system does not depend on the initial condition or on the affine term $F$ so that we can assume

$$
w(x, 0)=0, \quad w_{t}(x, 0)=0, \quad F(x, t)=0 .
$$

Controllability at time $T$ implies controllability at larger times so that: 
Definition 1.2. The sharp control time for system (1.1) (with control (1.2)) is the infimum of the set of the times at which the system is controllable.

The special case of equation (1.1) with $M(t) \equiv 0$ is the (generalized) telegrapher's equation:

$$
\left\{\begin{array}{l}
w_{t t}=2 c w_{t}+\nabla \cdot(a(x) \nabla w)+q(x) w, \\
w(x, t)=f(x, t) \quad x \in \Gamma, \quad w(x, t)=0, \quad x \in \partial \Omega \backslash \Gamma .
\end{array}\right.
$$

The paper [32] proves controllability of (1.4) with real controls if $\Gamma$ is suitably chosen and identifies a $\Gamma$-dependent control time. Our goal is the proof that, when the telegrapher's equation is controllable at time $T$, then also (1.1) is controllable and, conversely in the following sense:

Theorem 1.3. Let $\Omega \in \mathbb{R}^{3}$ be a bounded region with $C^{2}$ boundary and $M(t) \in H_{\mathrm{loc}}^{2}(0,+\infty), q(x) \in C(\bar{\Omega}), a(x) \in C^{1}(\bar{\Omega})$, with $a(x)>a_{0}>0$ for every $x \in \bar{\Omega}$. Then we have

(i) if the telegrapher's equation (1.4) is controllable at time $T$, then equation (1.1) is controllable at any larger time.

(ii) Equations (1.1) and (1.4) have the same sharp control time.

Among the different ways in which controllability can be proved, possibly the oldest one is the reduction of a control problem to a moment problem. Theorem 1.3, when $d=1$, has been proved via moment methods in $[\mathbf{1 9}, \mathbf{2 5}, \mathbf{2 6}]$, and we prove here that moment methods can be used in general.

As an application of our results, we note that controllability can be used to identify external signal using boundary observations, see [27]. Notations. Whenever the notations $\left\{M_{n}\right\}$ and $\left\{M_{n}(t)\right\}$ appear, they denote respectively a bounded sequence of numbers and a sequence of (continuous) functions which is bounded on a (preassigned) interval $[0, T]$, not the same sequences at every occurrence. The special expression of these sequences has no interest in the proofs.

We introduce the notation $(\partial / \partial \nu$ denotes normal derivative on $\partial \Omega)$

$$
G_{t}=\Gamma \times(0, t), \quad \gamma_{a} \phi=a(x) \frac{\partial \phi}{\partial \nu} \quad \text { on } \partial \Omega(\text { in particular on } \Gamma) .
$$


Organization of the paper is as follows. The goal of the paper is the proof of the two statements in Theorem 1.3. The proof is in two parts and requires several preliminaries and ancillary material. Preliminaries are in Section 2: subsection 2.1 presents a transformation of the variable $w$ which does not affect controllability but which simplifies the computations; subsection 2.2 presents information on the theory of moments and Riesz sequences, while the properties of the telegrapher's equation are in subsection 2.3.

In Section 3, we prove Theorem 1.3 (i) while equality of the sharp control times (i.e., item (ii)) is proved in Section 4.

Proofs of ancillary results are in the appendix.

1.1. References and known results. The first results on controllability of viscoelastic systems have been obtained by Leugering (see, for example, $[\mathbf{1 6}, \mathbf{1 7}]$ ) then followed by several contributions (see, for example, [15]). Among them, we consider in particular the results in $[\mathbf{5}, \mathbf{8}, \mathbf{2 0}, \mathbf{2 4}]$. The paper [20] proves Theorem 1.3 (even for a nonconvolution kernel. See [28] for an important special case) in the case $q(x)=0$ and $a(x)=1$. More importantly, it explicitly assumes that the control acts on the whole boundary of $\Omega, \Gamma=\partial \Omega$. Under these conditions, paper [20] proves controllability, as a consequence of observability of the adjoint system, when $T \geq T_{0}$, where $T_{0}$ is explicitly identified. Controllability via observability of the adjoint system is proved in [8], when the control is distributed in a subregion close to $\partial \Omega$ (the proof is based on Carleman estimates).

An extension of the D'Alembert formula is used in [5, Section 5] to study controllability to smooth targets of a (one-dimensional) thermal system with memory.

The paper [24] uses an operator approach and represents the solutions of (1.1) by using cosine operators (this idea is implicit in previous papers, for example by Leugering). It is proved in [24] that controllability holds for the equation with memory if the corresponding wave equation is controllable but the control time is not explicitly identified.

The papers $[\mathbf{5}, \mathbf{8}, \mathbf{2 4}]$ are concerned with the heat equation with memory so that they study only the controllability of the component $w(t)$, not of the velocity, but at least the arguments in [24] are easily extended to the pair (deformation/velocity). 
In conclusion, Theorem 1.3 extends and completes the results in $[5,8,20,24]$ and furthermore it uses different techniques, which have their independent interest: the proof uses moment methods and extends to spaces of higher dimension the techniques and results developed in $[1,3,4,19,25,26,27,28]$.

2. Preliminary information. Let $A$ be the operator in $L^{2}(\Omega)$,

$$
\operatorname{dom} A=H^{2}(\Omega) \cap H_{0}^{1}(\Omega), \quad A w=\nabla \cdot(a(x) \nabla w)+q(x) w .
$$

This operator is selfadjoint with compact resolvent and has a sequence $\left\{-\lambda_{n}^{2}\right\}$ of eigenvalues. Note the sign and the exponent, but this does not imply that $-\lambda_{n}^{2}$ is real negative. This property does depend on the sign of $q(x)$. The order of the eigenvalues is taken so that $\left\{\left|\lambda_{n}\right|\right\}$ is increasing (eigenvalues with equal modulus are taken in any order) and every eigenvalue is repeated according to its multiplicity (which is finite). It is known (see [23, page 192]) that there exist $N, m_{0}>0$ and $m_{1}>0$ such that, if $n>N$, then $\lambda_{n}^{2}$ is real, and we have:

$$
m_{0} n^{2 / d}<\lambda_{n}^{2}<m_{1} n^{2 / d} .
$$

We shall use the following consequence:

Lemma 2.1. If $d \leq 3$, then we have $\sum 1 / \lambda_{n}^{4}<+\infty$.

The space $L^{2}(\Omega)$ has an orthonormal basis whose elements are eigenvectors of $A$ : $A \phi_{n}=-\lambda_{n}^{2} \phi_{n}$.

For any $k>0$, such that $(k I-A)$ is positive, the sequence

$$
\left\{\phi_{n}\left(\sqrt{k+\lambda_{n}^{2}}\right)^{-1}\right\}
$$

is an orthonormal basis of $\left(\operatorname{dom}(k I-A)^{1 / 2}\right)$, and so $\left\{\phi_{n} \sqrt{k+\lambda_{n}^{2}}\right\}$ is an orthonormal basis of $\left(\operatorname{dom}(k I-A)^{1 / 2}\right)^{\prime}$. This space is unitarily equivalent to $H^{-1}(\Omega)$ since (from [9, Theorem 1-D]) (dom $(k I-$ $\left.A)^{1 / 2}\right)=H_{0}^{1}(\Omega)$. Hence, every $\chi \in H^{-1}(\Omega)$ has the representation

$$
\chi=\sum \chi_{n}\left(\sqrt{k+\lambda_{n}^{2}}\right) \phi_{n}, \quad\left\{\chi_{n}\right\} \in l^{2} .
$$


2.1. A preliminary transformation. The computations are simplified if we use a transformation first introduced in [25]. We integrate both sides of (1.1). Initial conditions and affine terms are zero, so that we get

$$
w_{t}(t)=2 c w(t)+\int_{0}^{t} \tilde{N}(t-s)(\nabla \cdot(a(x) \nabla w(s))+q(x) w(s)) \mathrm{d} s
$$

with

$$
\begin{aligned}
w(0) & =0, & & w_{\left.\right|_{\Gamma}}(t)=f(t), \\
w_{\left.\right|_{\text {} \Omega \backslash \Gamma}}(t) & =0, & \widetilde{N}(t) & =1+\int_{0}^{t} M(s) \mathrm{d} s .
\end{aligned}
$$

We introduce

$$
\theta(x, t)=e^{2 \gamma t} w(x, t), \quad \gamma=-M(0) / 2=-\tilde{N}^{\prime}(0) / 2 .
$$

We see that $\theta$ solves the following equation, where $\alpha=c+\gamma, N(t)=$ $e^{2 \gamma t} \tilde{N}(t)$ :

$$
\theta_{t}=2 \alpha \theta(t)+\int_{0}^{t} N(t-s)(\nabla \cdot(a(x) \nabla \theta(s))+q(x) \theta(s)) \mathrm{d} s
$$

with conditions

$$
\theta(0)=0 \quad \theta_{\left.\right|_{\Gamma}}(t)=e^{2 \gamma t} f(t), \quad \theta_{\left.\right|_{\partial \Omega \backslash \Gamma}}(t)=0
$$

(the functions $e^{2 \gamma t} f(t)$ will be renamed $f(t)$ ). The fact that simplifies the computation is:

$$
N(0)=1, \quad N^{\prime}(0)=0 .
$$

Thanks to the equality $w_{t}=e^{-2 \gamma t}\left(\theta_{t}-2 \gamma \theta\right)$, controllability of the pair $\left(w, w_{t}\right)$ is equivalent to controllability of the pair $\left(\theta, \theta_{t}\right)$. So, from now on, we study the controllability of the pairs $\left(\theta, \theta_{t}\right)$ where $\theta$ solves equation (2.3).

Now we compute the derivative of both sides of equation (2.3). We 
get

$$
\begin{aligned}
r \theta_{t t}= & 2 \alpha \theta_{t}+\nabla \cdot(a(x) \nabla \theta)+q(x) \theta \\
& +\int_{0}^{t} N(t-s)(\nabla \cdot(a(x) \nabla \theta(s))+q(x) \theta(s)) \mathrm{d} s .
\end{aligned}
$$

The telegrapher's equation associated to this system is

$$
w_{t t}=2 \alpha w_{t}+\nabla \cdot(a(x) \nabla w)+q(x) w
$$

(of course systems (2.5) and (1.4) have the same controllability properties).

We shall prove controllability of the viscoelastic system written in the form (2.3) by comparing it with the telegrapher's equation (2.5). In this study, the following notation will be of frequent use ( $\alpha$ is the coefficient in (2.3) and (2.5)):

$$
\beta_{n}=\sqrt{\lambda_{n}^{2}-\alpha^{2}}
$$

2.2. Riesz sequences and moment methods. The study of the controllability of linear systems can often be reduced to the solution of suitable moment problems. We confine ourselves to considering the special case which is needed in the proof of Theorem 1.3. Let $H$ be an infinite dimensional, separable (real or complex) Hilbert space (inner product is $\langle\cdot, \cdot\rangle$, and the norm is $|\cdot|)$. Let $\left\{e_{n}\right\}$ be a sequence in $H$. We define $\mathbb{J}: H \mapsto l^{2}$ as

$$
\operatorname{dom} \mathbb{J}=\left\{f \in H:\left\{\left\langle f, e_{n}\right\rangle\right\} \in l^{2}\right\}, \quad \mathbb{J} f=\left\{\left\langle f, e_{n}\right\rangle\right\} .
$$

The moment problem is the study of im $\mathbb{J}$. In particular, we are interested in understanding whether the sequence of the equations

$$
\left\langle f, e_{n}\right\rangle=c_{n}
$$

admits a solution $f$ for every $\left\{c_{n}\right\} \in l^{2}$, and to represent at least one of the solutions. In the proof of Theorem 1.3 we only use the case $\mathbb{J} \in \mathcal{L}\left(H, l^{2}\right)$. Then we restrict our interest to this case. It is then easy to compute $\mathbb{J}^{*}$ :

$$
\mathbb{J}^{*}\left(\left\{c_{n}\right\}\right)=\sum e_{n} c_{n}
$$

It turns out (see [2, Theorem I.2.1]) that $\mathbb{J}$ is an isomorphism of $\operatorname{clspan}\left\{e_{n}\right\}$ and $l^{2}$ if and only if $\left\{e_{n}\right\}$ is a Riesz sequence, i.e., if and 
only if $\left\{e_{n}\right\}$ is the image of an orthonormal basis of a Hilbert space $K$ under a linear bounded and boundedly invertible transformation from $K$ to $H$.

A Riesz sequence in $H$ which is complete in $H$ is called a Riesz basis. The following result holds (see [33, Theorem 9]):

Lemma 2.2. The sequence $\left\{e_{n}\right\}$ is a Riesz sequence if and only if there exist numbers $m_{0}>0$ and $m_{1}>0$ such that

$$
m_{0} \sum\left|a_{n}\right|^{2} \leq\left|\sum a_{n} e_{n}\right|_{H}^{2} \leq m_{1} \sum\left|a_{n}\right|^{2}
$$

for every finite sequence $\left\{a_{n}\right\}$. If, furthermore, the sequence $\left\{e_{n}\right\}$ is complete, then it is a Riesz basis.

Every Riesz sequence admits biorthogonal sequences $\left\{\psi_{n}\right\}$, i.e., sequences such that

$$
\left\langle\psi_{k}, e_{n}\right\rangle=\delta_{n, k}= \begin{cases}1 & \text { if } n=k \\ 0 & \text { if } n \neq k\end{cases}
$$

One (and only one) of these biorthogonal sequences belongs to the closed space spanned by $\left\{e_{n}\right\}$. This biorthogonal sequence is a Riesz sequence too, and the solution of the moment problem (2.7) is

$$
f=\sum c_{n} \psi_{n}
$$

Let $\left\{e_{n}\right\}$ and $\left\{z_{n}\right\}$ be two sequences in $H$. We say that they are quadratically close if

$$
\sum\left|e_{n}-z_{n}\right|^{2}<+\infty
$$

and we use the following test (see $[\mathbf{3 0}, \mathbf{3 3}])$ :

Theorem 2.3. Let $\left\{e_{n}\right\}$ be a Riesz sequence in $H$, and let $\left\{z_{n}\right\}$ be quadratically close to $\left\{e_{n}\right\}$. Then we have

- Paley-Wiener theorem. There exists $N$ such that $\left\{z_{n}\right\}_{n>N}$ is a Riesz sequence in $H$; 
- Bari theorem. The sequence $\left\{z_{n}\right\}$ is a Riesz sequence if, furthermore, it is $\omega$-independent, i.e., if (here $\left\{\alpha_{n}\right\}$ is a sequence of numbers)

$$
\sum \alpha_{n} z_{n}=0 \Longrightarrow\left\{\alpha_{n}\right\}=0 .
$$

A useful observation (implicitly used in the statement of Theorem 2.3) is as follows: if $\left\{z_{n}\right\}$ is quadratically close to a Riesz sequence, then $\sum \alpha_{n} z_{n}$ converges in $H$ if and only if $\left\{\alpha_{n}\right\} \in l^{2}$ (see [10, Chapter 6$])$.

The concrete case in which we are interested is the case $H=$ $L^{2}(0, T ; K)$ where $K$ is a second Hilbert space (it will be $K=L^{2}(\Gamma)$ ). In this context, we need two special results. For completeness, the proofs are given in the appendix.

Lemma 2.4. Let $\mathbb{Z}^{\prime}=\mathbb{Z} \backslash\{0\}$, and let $\left\{b_{n}\right\}_{n \in \mathbb{Z}^{\prime}},\left\{k_{n}\right\}_{n \in \mathbb{Z}^{\prime}}$ be such that

$$
b_{-n}=-b_{n}, \quad k_{n}=k_{-n} \in K, \quad\left|\mathcal{I} m b_{n}\right|<L
$$

for a suitable number $L$. If the sequence $\left\{e^{i b_{n} t} k_{n}\right\}_{n \in \mathbb{Z}^{\prime}}$ is a Riesz sequence in $L^{2}(0,2 T, K)$, then the following sequences are Riesz sequences in $L^{2}(0, T ; K)$ :

$$
\left\{k_{n} \cos b_{n} t\right\}_{n>0}, \quad\left\{k_{n} \sin b_{n} t\right\}_{n>0} .
$$

Now we consider a Riesz basis $\left\{e_{n}\right\}$ in $L^{2}(0, T ; K)$ and a time $T_{0}<T$. Then $\left\{e_{n}\right\}$ is complete in $L^{2}\left(0, T_{0} ; K\right)$, but it is not a Riesz sequence since every element of $L^{2}\left(0, T_{0} ; K\right)$ has infinitely many representations as a series $\sum a_{n} e_{n}$ (one such representation for every extension which belongs to $\left.L^{2}(0, T ; K)\right)$.

Let $\mathbb{J}_{0}$ be the operator from $L^{2}\left(0, T_{0} ; K\right)$ to $l^{2}$ given by

$$
\mathbb{J}_{0} f=\left\{\left\langle f, e_{n}\right\rangle_{L^{2}\left(0, T_{0} ; K\right)}\right\} .
$$

We prove:

Lemma 2.5. $\operatorname{dim}\left[\operatorname{im} \mathbb{J}_{0}\right]^{\perp}=+\infty$. 
Finally, we note that $(2.2)$ can be written as $\left(\beta_{n}\right.$ is defined in $\left.(2.6)\right)$ :

$$
\chi=\sum_{\lambda_{n}^{2}=\alpha^{2}}\left(\chi_{n} \sqrt{k+\alpha^{2}}\right) \phi_{n}+\sum_{\lambda_{n}^{2} \neq \alpha^{2}}\left(\frac{\chi_{n} \sqrt{k+\lambda_{n}^{2}}}{\beta_{n}}\right)\left[\beta_{n} \phi_{n}\right] .
$$

It follows that a Riesz basis of $H^{-1}(\Omega)$ is the sequence whose elements are

$$
\begin{cases}\phi_{n} & \text { if } \lambda_{n}^{2}=\alpha^{2} \\ \beta_{n} \phi_{n} & \text { if } \lambda_{n}^{2} \neq \alpha^{2}\end{cases}
$$

2.3. The telegrapher's equation. We consider the telegrapher's equation (2.5) associated to equation (2.4). Controllability at time $T$ is equivalent to surjectivity of the map $f \mapsto \Lambda_{T} f=\left(w(T), w_{t}(T)\right)$ (from $L^{2}\left(G_{T}\right)$ to $L^{2}(\Omega) \times H^{-1}(\Omega)$ ). By computing $\Lambda_{T}^{*}$, we see that the telegrapher's equation is controllable at time $T$ if and only if there exist $m=m_{T}>0, M=M_{T}>0$ such that

$$
\begin{aligned}
m\left(\left\|\phi_{0}\right\|_{H_{0}^{1}(\Omega)}^{2}+\left\|\phi_{1}\right\|_{L^{2}(\Omega)}^{2}\right) & \leq \int_{G_{T}}\left\|\gamma_{a} \phi\right\|^{2} \mathrm{~d} G_{T} \\
& \leq M\left(\left\|\phi_{0}\right\|_{H_{0}^{1}(\Omega)}^{2}+\left\|\phi_{1}\right\|_{L^{2}(\Omega)}^{2}\right)
\end{aligned}
$$

Here $\phi$ denotes the solution of the adjoint system

$$
\begin{aligned}
\phi_{t t} & =-2 \alpha \phi_{t}+\nabla \cdot(a(x) \nabla \phi)+q(x) \phi \\
\phi(\cdot, 0) & =\phi_{0}(x) \in H_{0}^{1}(\Omega), \quad \phi_{t}(\cdot, 0)=\phi_{1}(x) \in L^{2}(\Omega), \phi_{\left.\right|_{\partial \Omega}}=0 .
\end{aligned}
$$

The inequalities (2.13) have the following consequence:

Theorem 2.6. Let $T>0$, and let the telegrapher's equation (2.5) be controllable at time $T$. Then we have:

(i) For every target $\left.(\xi, \eta) \in L^{2}(\Omega) \times H^{-1}(\Omega)\right)$, there exists a unique steering control $f=f^{(\xi, \eta)} \in L^{2}\left(G_{T}\right)$ of minimal norm. This steering control is a continuous function of $(\xi, \eta)$.

(ii) Let $\phi(x)$ be an eigenvector of $A$. Then $\int_{\Gamma}\left|\gamma_{a} \phi\right|^{2} \mathrm{~d} \Gamma \neq 0$.

(iii) The sequence $\left\{\left(\gamma_{a} \phi_{n}\right) / \lambda_{n}\right\}_{\lambda_{n} \neq 0}$ is almost normalized in $L^{2}(\Gamma)$, i.e., there exist $m>0$ and $M$ such that

$$
0<m \leq\left\|\left(\gamma_{a} \phi_{n}\right) / \lambda_{n}\right\|_{L^{2}(\Gamma)} \leq M
$$


Proof. Statement (i) follows since the left inequality in (2.13) is coercivity of the adjoint of the map $f \mapsto\left(w(T), w^{\prime}(T)\right)$ (see $\left.[\mathbf{1 8}, \mathbf{2 2}]\right)$.

We prove statement (ii). Let $A \phi=\lambda \phi$. If $\beta=\sqrt{\lambda^{2}-\alpha^{2}} \neq 0$, then the function $\phi(x, t)=e^{-\alpha t} \phi(x) \sin \beta t$ solves (2.14). The left inequality in (2.13) shows that

$$
m \beta^{2} \|\left.\phi\right|_{L^{2}(\Omega)} ^{2} \leq\left[\int_{0}^{T} e^{-2 \alpha t} \sin ^{2} \beta t \mathrm{~d} t\right] \int_{\gamma}\left|\gamma_{a} \phi(x)\right|^{2} \mathrm{~d} \Gamma .
$$

The result follows since (by definition) the eigenvectors are nonzero.

If $\beta=0$, a similar argument holds, with $\phi(x, t)=e^{-\alpha t} \phi(x)$.

We prove statement (iii) (see [13] for the idea of the proof). Let $\beta_{n}=\sqrt{\lambda_{n}^{2}-\alpha^{2}}$. It may be $\beta_{n}=0$ in (2.6) only for a finite set of indices. So, in the proof of the asymptotic estimate (2.15), we can assume $\beta_{n}=\sqrt{\lambda_{n}^{2}-\alpha^{2}} \neq 0$.

The function $\phi(x, t)=1 / \beta_{n} e^{-\alpha t} \phi_{n}(x) \sin \beta_{n} t$ solves equation (2.14) with initial conditions

$$
\phi(x, 0)=0, \quad \phi_{t}(x, 0)=\phi_{n}(x) .
$$

By using $\left\|\phi_{n}\right\|_{L^{2}(\Omega)}=1$, inequality (2.13) gives

$$
m \leq\left[\int_{0}^{T}\left(\frac{\lambda_{n}}{\beta_{n}} e^{-\alpha t} \sin \beta_{n} t\right)^{2} \mathrm{~d} t\right] \int_{\Gamma}\left|\frac{\gamma_{a} \phi_{n}}{\lambda_{n}}\right|^{2} \mathrm{~d} \Gamma<M .
$$

The result follows since $\lim _{n \rightarrow+\infty}\left(\lambda_{n} / \beta_{n}\right)=1$ and

$$
\begin{aligned}
\lim _{n \rightarrow+\infty} \int_{0}^{T} e^{-2 \alpha t} \sin ^{2} \beta_{n} t \mathrm{~d} t & =\frac{\left(1-e^{-2 \alpha T}\right)}{4 \alpha}, \\
\lim _{n \rightarrow+\infty} \int_{0}^{T} \sin ^{2} \beta_{n} t \mathrm{~d} t & =\frac{1}{2} T .
\end{aligned}
$$

2.3.1. Moment method for the telegrapher's equation. The following computations make sense for smooth controls and are then extended to square integrable controls by continuity. Let $\left(\phi_{n}\right.$ are eigenvectors of A)

$$
w_{n}(t)=\int_{\Omega} w(x, t) \phi_{n}(x) \mathrm{d} x
$$


Then, $w_{n}(t)$ solves

$$
w_{n}^{\prime \prime}=2 \alpha w_{n}^{\prime}-\lambda_{n}^{2} w_{n}-\int_{\Gamma}\left(\gamma_{a} \phi_{n}\right) f(x, t) \mathrm{d} \Gamma .
$$

So, with $\beta_{n}$ defined in (2.6), we have

$$
\begin{array}{ll}
w_{n}(t)=-\int_{G_{t}} e^{\alpha s}\left[\frac{\gamma_{a} \phi_{n}}{\beta_{n}} \sin \beta_{n} s\right], & \beta_{n} \neq 0 \\
w_{n}(t)=-\int_{G_{t}} s e^{\alpha s}\left[\gamma_{a} \phi_{n}\right] f(x, t-s) \mathrm{d} G_{t}, & \beta_{n}=0
\end{array}
$$

(it may be $\beta_{n}=0$ for a finite number of indices). So, we have

$$
\begin{aligned}
-w(x, t) & =\sum \phi_{n}(x) \int_{G_{t}} e^{\alpha s}\left[\frac{\gamma_{a} \phi_{n}}{\beta_{n}} \sin \beta_{n} s\right] f(x, t-s) \mathrm{d} G_{t} \\
-w_{t}(x, t) & =\sum \beta_{n} \phi_{n}(x) \\
& \cdot \int_{G_{t}} e^{\alpha s} \frac{\gamma_{a} \phi_{n}}{\beta_{n}}\left[\frac{\alpha}{\beta_{n}} \sin \beta_{n} s+\cos \beta_{n} s\right] f(x, t-s) \mathrm{d} G_{t} .
\end{aligned}
$$

If $\beta_{n}=0$, then the corresponding term in (2.18) is replaced with (2.17), while in (2.19) it is replaced with

$$
\int_{G_{t}}(1+\alpha s) e^{\alpha s}\left(\gamma_{a} \phi_{n}\right) f(x, t-s) \mathrm{d} G_{t} .
$$

Equalities (2.18) and (2.19) show that controllability at time $T$ of the telegrapher's equation is equivalent to solvability of the following moment problem

$$
\begin{aligned}
& \int_{G_{T}} e^{\alpha s}\left[\frac{\gamma_{a} \phi_{n}}{\beta_{n}} \sin \beta_{n} s\right] f(x, T-s) \mathrm{d} G_{T}=\xi_{n} \\
& \int_{G_{T}} e^{\alpha s} \frac{\gamma_{a} \phi_{n}}{\beta_{n}}\left[\frac{\alpha}{\beta_{n}} \sin \beta_{n} s+\cos \beta_{n} s\right] f(x, T-s) \mathrm{d} G_{T}=\eta_{n}
\end{aligned}
$$

where $\left\{\xi_{n}\right\}$ and $\left\{\eta_{n}\right\}$ belong to $l^{2}$ and $f$ is real.

We noted that, when $\beta_{n}=0$, the corresponding terms in (2.21) and (2.22) have to be replaced respectively with (2.17) or (2.20). In order to have a unified formulation, we introduce

$$
\mathcal{J}=\left\{n: \beta_{n}=0\right\}
$$


(a finite set of indices) and $c_{n}=\eta_{n}+i \xi_{n}$. Then, $\left\{c_{n}\right\}$ is an arbitrary (complex valued) $l^{2}$ sequence. The moment problem (2.21)-(2.22) reduces to the following problem where $g(x, s)=e^{\alpha s} f(x, T-s)$ is real:

$$
\begin{aligned}
& \left\langle g, \bar{e}_{n}\right\rangle=\int_{G_{T}} e_{n}(x, s) g(s) \mathrm{d} s=c_{n}, \quad n>0 \\
& e_{n}(x, s)= \begin{cases}\left(\frac{\gamma_{a} \phi_{n}}{\beta_{n}}\right)\left[e^{i \beta_{n} s}+\frac{\alpha}{\beta_{n}} \sin \beta_{n} s\right], & n \notin \mathcal{J} \\
(1+\alpha s+i s)\left(\gamma_{a} \phi_{n}\right), & n \in \mathcal{J} .\end{cases}
\end{aligned}
$$

Statement (i) in Theorem 2.6 is equivalent to the following fact: for every target $(\xi, \eta) \in L^{2}(\Omega) \times H^{-1}(\Omega)$ there exists a real steering control $f$, which depends continuously on $(\xi, \eta)$.

The index $n$ in (2.23) is positive. It is convenient to reformulate the problem with $n \in \mathbb{Z}^{\prime}=\mathbb{Z} \backslash\{0\}$. We define, for $n<0$ and $-n \notin \mathcal{J}$ :

$$
\beta_{n}=-\left(\bar{\beta}_{-n}\right), \quad \phi_{n}=\phi_{-n}, \quad \lambda_{n}=\lambda_{-n} .
$$

This implies $e_{-n}=-\bar{e}_{n}$ if $-n \notin \mathcal{J}$, and this is the definition of $e_{-n}$ for $-n \in \mathcal{J}$. A real solution $f$ of problem (2.23) (and $n>0$ ) exists and depends continuously on the complex sequence $\left\{c_{n}\right\}_{n>0} \in l^{2}$ if and only if the moment problem

$$
\left\langle f, \bar{e}_{n}\right\rangle_{L^{2}\left(G_{T}\right)}=c_{n}, \quad n \in \mathbb{Z}^{\prime}
$$

admits a complex valued solution $f \in L^{2}\left(G_{T}\right)$ which depends continuously on $(\xi, \eta)$ (the proof is the same as in the memory case, and it is given in the appendix). That is, controllability of the telegrapher's equation is equivalent to the fact that the moment operator of the sequence $\left\{\bar{e}_{n}\right\}$ is bounded and boundedly invertible. The sequences $\left\{e_{n}\right\}$ and $\left\{\bar{e}_{n}\right\}$ have the same basis properties. So we can state:

Theorem 2.7. The telegrapher's equation is controllable in time $T$ if and only if the sequence $\left\{e_{n}\right\}_{n \in \mathbb{Z}^{\prime}}$ is a Riesz sequence in $L^{2}\left(G_{T}\right)$ (with complex scalars).

It is interesting to note that, when $\left\{\xi_{n}\right\}$ and $\left\{\eta_{n}\right\}$ are arbitrary in $l^{2}$, the same holds for the sequence $\left\{\eta_{n}+i \xi_{n}-\left(\gamma / \beta_{n}\right) \xi_{n}\right\}$, for every number $\gamma$. So, Theorem 2.7 holds as well if the functions $e_{n}$ in (2.23) 
are replaced by

$$
\begin{gathered}
\left(e^{i \beta_{n} t}+\frac{\gamma}{\beta_{n}} \sin \beta_{n} t\right) \frac{\gamma_{a} \phi_{n}}{\beta_{n}}, \quad n \notin \mathcal{J}, \\
(1+(i+\gamma) s) \gamma_{a} \phi_{n}, \quad n \in \mathcal{J}
\end{gathered}
$$

where $\gamma$ is any fixed complex number (possibly $\gamma=0$ ).

3. Controllability of the viscoelastic systems. In this section, we prove item (i) of Theorem 1.3. Let $\theta$ solve (2.4)) and

$$
\theta_{n}(t)=\int_{\Omega} \theta(x, t) \phi_{n}(x) \mathrm{d} x .
$$

Then we have

$\theta_{n}^{\prime}=2 \alpha \theta_{n}-\lambda_{n}^{2} \int_{0}^{t} N(t-s) \theta_{n}(s) \mathrm{d} s-\int_{0}^{t} N(t-s)\left[\int_{\Gamma}\left(\gamma_{a} \phi_{n}\right) f(x, s) \mathrm{d} \Gamma\right] \mathrm{d} s$.

For every $n$, we introduce the functions $z_{n}(t)$ which solve

$$
z_{n}^{\prime}=2 \alpha z_{n}-\lambda_{n}^{2} \int_{0}^{t} N(t-s) z_{n}(s) \mathrm{d} s, \quad z_{n}(0)=1 .
$$

Hence:

$$
\begin{aligned}
\theta_{n}(t) & =-\int_{0}^{t} z_{n}(\tau) \int_{0}^{t-\tau} N(t-\tau-s) \int_{\Gamma}\left(\gamma_{a} \phi_{n}\right) f(x, s) \mathrm{d} \Gamma \mathrm{d} s \mathrm{~d} \tau \\
& =-\int_{G_{t}}\left\{\int_{0}^{s} N(s-\tau) z_{n}(\tau) \mathrm{d} \tau\right\}\left(\gamma_{a} \phi_{n}\right) f(x, t-s) \mathrm{d} G_{t}
\end{aligned}
$$

$$
\theta_{n}^{\prime}(t)=-\int_{G_{t}}\left[z_{n}(s)+\int_{0}^{s} N^{\prime}(s-\tau) z_{n}(\tau) \mathrm{d} \tau\right]\left(\gamma_{a} \phi_{n}\right) f(x, t-s) \mathrm{d} G_{t} .
$$

Thanks to continuous dependence of the solutions on the initial data and regularity when the initial data are smooth (see [14] and [24, Appendix $])$ ), the following equalities hold in $C\left([0, T] ; L^{2}(\Omega)\right)$ and $C\left([0, T] ; H^{-1}(\Omega)\right)$ :

$$
\theta(t)=\sum_{n=1}^{+\infty} \theta_{n}(t) \phi_{n}(x), \quad \theta_{t}(t)=\sum_{n=1}^{+\infty} \theta_{n}^{\prime}(t) \phi_{n}(x)
$$


Let $\left\{\xi_{n}\right\} \in l^{2}$ and $\left\{\eta_{n}\right\} \in l^{2}$ be the sequence of the coefficients of the expansions of the targets $\xi$ and $\eta$ in series of, respectively, $\left\{\phi_{n}\right\}$ and $\left\{\beta_{n} \phi_{n}\right\}\left(\beta_{n} \phi_{n}\right.$ replaced with $\phi_{n}$ if $\left.\beta_{n}=0\right)$. We see that controllability at time $T$ is equivalent to the solvability of the following moment problem:

$$
\begin{array}{ll}
\int_{G_{T}} Z_{n}(t) \frac{\gamma_{a} \phi_{n}}{\beta_{n}} f(x, T-s) \mathrm{d} G_{T}=c_{n}=-\left(\eta_{n}+i \xi_{n}\right), & n \notin \mathcal{J} \\
\int_{G_{T}} Z_{n}(t)\left(\gamma_{a} \phi_{n}\right) f(x, T-s) \mathrm{d} G_{T}=c_{n}=-\left(\eta_{n}+i \xi_{n}\right), \quad n \in \mathcal{J}
\end{array}
$$

where, for $n>0$,

$$
Z_{n}(t)=\left\{\begin{array}{rr}
z_{n}(t)+\int_{0}^{t} N^{\prime}(t-s) z_{n}(s) \mathrm{d} s & \\
& +i \beta_{n} \int_{0}^{t} N(t-s) z_{n}(s) \mathrm{d} s, \quad n \notin \mathcal{J}, \\
z_{n}(t)+\int_{0}^{t} N^{\prime}(t-s) z_{n}(s) \mathrm{d} s & \\
+i \int_{0}^{t} N(t-s) z_{n}(s) \mathrm{d} s, & n \in \mathcal{J}
\end{array}\right.
$$

(we recall that if $n \in \mathcal{J}$ then the element $\beta_{n} \phi_{n}$ of the basis of $H^{-1}(\Omega)$ has to be replaced with $\left.\phi_{n}\right)$.

It is convenient to reformulate the moment problem with $n \in \mathbb{Z}^{\prime}$. This is done by using the following definitions:

$$
\begin{aligned}
z_{-n}(t) & =z_{n}(t), & & \phi_{-n}(x)=\phi_{n}(x), \\
\beta_{-n} & =-\bar{\beta}_{n}, & & \lambda_{-n}=\lambda_{n}, \quad n \notin \mathcal{J} .
\end{aligned}
$$

Let

$$
\Psi_{n}=\frac{\gamma_{a} \phi_{n}}{\beta_{n}}, \quad n \notin \mathcal{J}, \quad \Psi_{n}=\gamma_{a} \phi_{n}, \quad n \in \mathcal{J} .
$$

Then we have

$$
Z_{-n} \Psi_{-n}=-\bar{Z}_{n} \bar{\Psi}_{n}, \quad n \notin \mathcal{J} .
$$

We symmetrize $\mathcal{J}$ (with respect to 0 ) and we define $Z_{-n} \Psi_{-n}$ when $n \in \mathcal{J}$ by:

$$
Z_{-n} \Psi_{-n}=-\bar{Z}_{n} \bar{\Psi}_{n}=-\bar{Z}_{n} \Psi_{n} \quad \text { if } n \in \mathcal{J}
$$

So, we can consider the moment problem (3.4) with $n \in \mathbb{Z}^{\prime}=\mathbb{Z} \backslash\{0\}$. 
It is proved in the Appendix that $\left\{Z_{n} \Psi_{n}\right\}_{n \in \mathbb{Z}^{\prime}}$ is a Riesz sequence if and only if the moment problem (3.4) (with $n>0$ ) admits a real solution $f$ which is a continuous function of $\left(\eta_{n}+i \xi_{n}\right) \in l^{2}$. So, in order to prove the first statement in Theorem 1.3, we prove:

Theorem 3.1. Let the telegrapher's equation (2.5) be controllable at time $T-\varepsilon$. Then, the sequence $\left\{Z_{n}(t) \Psi_{n}\right\}_{n \in \mathbb{Z}^{\prime}}$ is a Riesz sequence in $L^{2}\left(G_{T}\right)$.

The proof of Theorem 3.1 is in two steps: we prove that $\left\{Z_{n}(t) \Psi_{n}\right\}$ is quadratically close to a Riesz sequence and then we prove that it is $\omega$-independent.

\subsection{Step 1: Closeness to a Riesz sequence. Let}

$$
\begin{aligned}
& K_{n}(t)=N^{\prime}(t)+i \beta_{n} N(t) \quad \text { if } n \notin \mathcal{J}, \\
& K_{n}(t)=N^{\prime}(t)+i N(t) \quad \text { if } n \in \mathcal{J} .
\end{aligned}
$$

The right hand side of the equality (3.5) is a variation of constants formula, so that (compare (3.1)) $Z_{n}(t)$ solves

$$
Z_{n}^{\prime}=2 \alpha Z_{n}-\lambda_{n}^{2} \int_{0}^{t} N(t-s) Z_{n}(s) \mathrm{d} s+K_{n}(t), \quad Z_{n}(0)=1 .
$$

Hence, also

$$
\begin{aligned}
& Z_{n}^{\prime \prime}=2 \alpha Z_{n}^{\prime}-\lambda_{n}^{2} Z_{n}-\lambda_{n}^{2} \int_{0}^{t} N^{\prime}(t-s) Z_{n}(s) \mathrm{d} s+K_{n}^{\prime}(t), \\
& \left\{\begin{array}{l}
Z_{n}(0)=1, \\
Z_{n}^{\prime}(0)=2 \alpha+i \beta_{n}(n \notin \mathcal{J}), \quad Z_{n}^{\prime}(0)=2 \alpha+i \quad(n \in \mathcal{J}) .
\end{array}\right.
\end{aligned}
$$

Then we have the following representation formulas:

if $n \notin \mathcal{J}$ then,

$$
\begin{aligned}
& Z_{n}(t)=e^{\alpha t} e^{i \beta_{n} t}+e^{\alpha t} \frac{\alpha}{\beta_{n}} \sin \beta_{n} t \\
& +\frac{1}{\beta_{n}} \int_{0}^{t} e^{\alpha(t-s)} \sin \beta_{n}(t-s)\left[K_{n}^{\prime}(s)-\lambda_{n}^{2} \int_{0}^{s} N^{\prime}(s-r) Z_{n}(r) \mathrm{d} r\right] \mathrm{d} s,
\end{aligned}
$$

if $n \in \mathcal{J}$ then,

$$
Z_{n}(t)=e^{\alpha t}(1+(\alpha+i) t)
$$




$$
+\int_{0}^{t} e^{\alpha(t-s)}(t-s)\left[\left(N^{\prime \prime}(s)+i N^{\prime}(s)\right)-\alpha^{2} \int_{0}^{r} N^{\prime}(r-s) Z_{n}(s) \mathrm{d} s\right] \mathrm{d} r .
$$

We introduce

$$
S_{n}(t)=e^{-\alpha t} Z_{n}(t)
$$

and we see that, for $n \notin \mathcal{J}$,

$$
S_{n}(t)=G_{n}(t)-\frac{\lambda_{n}^{2}}{\beta_{n}} \int_{0}^{t} \sin \beta_{n}(t-s) \int_{0}^{s}\left(e^{-\alpha(s-r)} N^{\prime}(s-r)\right) S_{n}(r) \mathrm{d} r \mathrm{~d} s
$$

where (in the last integration by parts we use $N^{\prime}(0)=0$ ).

$$
\begin{aligned}
G_{n}(t)= & e^{i \beta_{n} t}+\frac{\alpha}{\beta_{n}} \sin \beta_{n} t \\
& +\frac{1}{\beta_{n}} \int_{0}^{t} e^{-\alpha s}\left[N^{\prime \prime}(s)+i \beta_{n} N^{\prime}(s)\right] \sin \beta_{n}(t-s) \mathrm{d} s \\
= & e^{i \beta_{n} t}+\frac{\alpha-N^{\prime}(0)}{\beta_{n}} \sin \beta_{n} t \\
& +\int_{0}^{t} N^{\prime}(t-s) e^{-\alpha(t-s)}\left[e^{i \beta_{n}(t-s)}+\frac{\alpha}{\beta_{n}} \sin \beta_{n}(t-s)\right] \mathrm{d} s \\
= & e^{i \beta_{n} t}+\frac{\alpha}{\beta_{n}} \sin \beta_{n} t \\
& +\int_{0}^{t} N^{\prime}(t-s) e^{-\alpha(t-s)}\left(e^{i \beta_{n} s}+\frac{\alpha}{\beta_{n}} \sin \beta_{n} s\right) \mathrm{d} s
\end{aligned}
$$

Instead, for $n \in \mathcal{J}$, we have

$$
G_{n}(t)=1+(\alpha+i) t+\int_{0}^{t} e^{-\alpha(t-s)} N^{\prime}(t-s)[1+(\alpha+i) s] \mathrm{d} s .
$$

The linear transformation

$$
y \longmapsto y(t)+\int_{0}^{t} e^{-\alpha(t-s)} N^{\prime}(t-s) y(s) \mathrm{d} s
$$

is bounded with bounded inverse. So, Theorem 2.7 and controllability of the telegrapher's equation (2.5) imply that the sequence $\left\{G_{n}(t) \Psi_{n}\right\}$ is Riesz in $L^{2}\left(G_{T}\right)$.

We shall need asymptotic estimates of $S_{n}(t)$ which hold for large $n$. So we can work with $n \notin \mathcal{J}$. We introduce the notation

$$
N_{1}(t)=e^{-\alpha t} N^{\prime}(t) \quad \text { so that } N_{1}(0)=0,
$$




$$
\mu_{n}=\frac{\lambda_{n}^{2}}{\beta_{n}^{2}} \quad \text { so that } 1-\mu_{n}=-\alpha^{2} / \beta_{n}^{2}
$$

An integration by parts gives

$$
\begin{aligned}
S_{n}(t)= & G_{n}(t)-\mu_{n} \int_{0}^{t} N_{1}(t-r) S_{n}(r) \mathrm{d} r \\
& +\mu_{n} \int_{0}^{t}\left(\int_{0}^{t-r} N_{1}^{\prime}(t-r-s) \cos \beta_{n} s \mathrm{~d} s\right) S_{n}(r) \mathrm{d} r .
\end{aligned}
$$

The Gronwall inequality shows that, for every $T>0$, there exists $M=M_{T}$ such that

$$
\left|S_{n}(t)\right| \leq M, \quad t \in[0, T]
$$

We integrate the last integral in (3.10) by parts again, and we get

$$
\begin{aligned}
S_{n}(t)= & G_{n}(t)-\mu_{n} \int_{0}^{t} N_{1}(t-r) S_{n}(r) \mathrm{d} r \\
& +\frac{\mu_{n}}{\beta_{n}} \int_{0}^{t}\left(N_{1}^{\prime}(0) \sin \beta_{n}(t-r)\right. \\
& \left.+\int_{0}^{t-r} N_{1}^{\prime \prime}(t-r-s) \sin \beta_{n} s \mathrm{~d} s\right) S_{n}(r) \mathrm{d} r .
\end{aligned}
$$

We introduce

$$
E_{n}(t)=e^{i \beta_{n} t}+\frac{\alpha}{\beta_{n}} \sin \beta_{n} t \quad(\text { if } n \notin \mathcal{J}),
$$

and we rewrite $(3.11)$ as ( $\star$ denotes the convolution)

$$
\left(S_{n}-E_{n}\right)+N_{1} \star\left(S_{n}-E_{n}\right)=\frac{N_{1}^{\prime}(0)}{\beta_{n}} \int_{0}^{t} \sin \beta_{n}(t-r) S_{n}(r) \mathrm{d} r
$$

$$
\begin{aligned}
+ & \frac{1}{\beta_{n}} \int_{0}^{t} N_{1}^{\prime \prime}(s) \int_{0}^{t-s} \sin \beta_{n}(t-s-r) S_{n}(r) \mathrm{d} r \mathrm{~d} s+\frac{1}{\beta_{n}{ }^{2}} M_{n}(t), \\
M_{n}(t) & =-\alpha^{2} \int_{0}^{t} N_{1}(t-r) S_{n}(r) \mathrm{d} r \\
& +\frac{\alpha}{\beta_{n}} \int_{0}^{t}\left[N_{1}^{\prime}(0) \sin \beta_{n}(t-r)+\int_{0}^{t-r} N_{1}^{\prime \prime}(t-r-s) \sin \beta_{n} s \mathrm{~d} s\right] \mathrm{d} r .
\end{aligned}
$$


Note that here we have explicitly written the expression of the functions $M_{n}(t)$, but this expression does not have a real interest: the important fact is that the sequence $\left\{M_{n}(t)\right\}$ is bounded on (any) interval $[0, T]$. This is the sole property of interest and, as we said already, in the following we use $\left\{M_{n}(t)\right\}$ to denote a sequence of (continuous) functions which is bounded (on an interval $[0, T]$ ), not the same sequence at every occurrence. We shall not write down the explicit expression of the functions $M_{n}(t)$, which has no role in the proofs.

By using the definition of $E_{n}(t)$, we see the existence of a sequence $\left\{M_{n}(t)\right\}$ of continuous functions defined for $t \geq 0$, bounded on bounded intervals and such that

$$
S_{n}(t)=e^{i \beta_{n} t}+\frac{M_{n}(t)}{\beta_{n}} .
$$

Now we compute:

$$
\int_{0}^{t} S_{n}(r) \sin \beta_{n}(t-r) \mathrm{d} r
$$

$$
\begin{aligned}
= & \int_{0}^{t}\left(e^{i \beta_{n} r}+\frac{M_{n}(r)}{\beta_{n}}\right) \sin \beta_{n}(t-r) \mathrm{d} r=-\frac{i}{2} t e^{i \beta_{n} t}+\frac{i}{2 \beta_{n}} \sin \beta_{n} t \\
& +\frac{1}{\beta_{n}} \int_{0}^{t} M_{n}(r) \sin \beta_{n}(t-r) \mathrm{d} r .
\end{aligned}
$$

We observe

$$
\begin{aligned}
\frac{i}{\beta_{n}} t e^{i \beta_{n} t} & =-\int_{0}^{t} s e^{i \beta_{n} s} \mathrm{~d} s+\frac{1}{\beta_{n}{ }^{2}}\left(e^{i \beta_{n} t}-1\right) \\
& =-\int_{0}^{t} s E_{n}(s) \mathrm{d} s+\frac{1}{{\beta_{n}}^{2}} M_{n}(t) .
\end{aligned}
$$

i.e.,

$$
\begin{aligned}
\frac{1}{\beta_{n}} \int_{0}^{t} S_{n}(r) \sin \beta_{n}(t-r) \mathrm{d} r & =-\frac{1}{2} \frac{i t}{\beta_{n}} e^{i \beta_{n} t}+\frac{1}{{\beta_{n}}^{2}} M_{n}(t) \\
& =\frac{1}{2} \int_{0}^{t} s E_{n}(s) \mathrm{d} s+\frac{1}{\beta_{n}{ }^{2}} M_{n}(t) .
\end{aligned}
$$


We replace this expression in (3.12) and we rewrite the equality as

$$
\begin{aligned}
\left(S_{n}-E_{n}\right)+N_{1} \star\left(S_{n}-E_{n}\right)= & \frac{N_{1}^{\prime}(0)}{2} \int_{0}^{t} s E_{n}(s) \mathrm{d} s \\
& +\frac{1}{2} \int_{0}^{t} N_{1}^{\prime \prime}(s) \int_{0}^{t-s} r E_{n}(r) \mathrm{d} r \mathrm{~d} s \\
& +\frac{1}{\beta_{n}^{2}} M_{n}(t) \\
= & \frac{1}{2} \int_{0}^{t} N_{1}^{\prime}(t-r) r E_{n}(r) \mathrm{d} r+\frac{1}{\beta_{n}{ }^{2}} M_{n}(t)
\end{aligned}
$$

(as usual, the functions $M_{n}(t)$ are not the same at every step).

Let $L(t)$ be the resolvent kernel of $N_{1}(t)$ so that $L(0)=0$ and $L(t)$ is twice differentiable. We have

$$
\begin{aligned}
S_{n}(t)= & E_{n}(t)+\frac{1}{2} \int_{0}^{t} N_{1}^{\prime}(t-s) s E_{n}(s) \mathrm{d} s \\
& -\frac{1}{2} \int_{0}^{t}\left(s E_{n}(s)\right)\left[\int_{0}^{t-s} L(t-s-r) N_{1}^{\prime}(r) \mathrm{d} r\right] \mathrm{d} s \\
& +\frac{1}{\beta_{n}^{2}} M_{n}(t) .
\end{aligned}
$$

In conclusion,

$$
\begin{aligned}
\Psi_{n} S_{n}(t)= & \Psi_{n} E_{n}(t) \\
& +\frac{1}{2} \int_{0}^{t} s\left[N_{1}^{\prime}(t-s)-\int_{0}^{t-s} L(t-s-r) N_{1}^{\prime}(r) \mathrm{d} r\right] \Psi_{n} E_{n}(s) \mathrm{d} s \\
& +\frac{1}{\beta_{n}{ }^{2}} M_{n}(t)
\end{aligned}
$$

(note that we can replace $\Psi_{n} M_{n}(t)$ with $M_{n}(t)$ since $\left\{\Psi_{n}\right\}$ is bounded in $\left.L^{2}(\Gamma)\right)$. The sequence whose elements are

$$
\Psi_{n} E_{n}(t)+\frac{1}{2} \int_{0}^{t} s\left[N_{1}^{\prime}(t-s)-\int_{0}^{t-s} L(t-s-r) N_{1}^{\prime}(r) \mathrm{d} r\right] \Psi_{n} E_{n}(s) \mathrm{d} s
$$

is the image of a Riesz sequence of $L^{2}\left(G_{T}\right)$ under a linear bounded and boundedly invertible transformation. Hence, it is a Riesz sequence too so that, by using Theorem 2.3 and Lemma 2.1, we get: 
Theorem 3.2. Let the telegrapher's equation (2.5) be controllable at time $T$. Then, $\left\{S_{n}(t) \Psi_{n}\right\}_{n \in \mathbb{Z}^{\prime}}$ is quadratically close to a Riesz sequence in $L^{2}\left(G_{T}\right)$ and so there exists $N$ such that $\left\{S_{n}(t) \Psi_{n}\right\}_{|n|>N}$ is a Riesz sequence too.

In the second step, we prove that $\left\{S_{n}(t) \Psi_{n}\right\}_{n \in \mathbb{Z}^{\prime}}$ is $\omega$-independent in $L^{2}\left(G_{T}\right)$; hence, it is a Riesz sequence and this completes the proof of statement (i) in Theorem 1.3.

3.2. Step 2: $\omega$-independence. We consider the equality

$$
\sum_{n \neq 0} \alpha_{n} S_{n}(t) \Psi_{n}=0 \quad \text { in } L^{2}\left(G_{T}\right) .
$$

Theorem 3.2 implies that $\left\{\alpha_{n}\right\} \in l^{2}$. Our goal is the proof that $\alpha_{n}=0$ for every $n$. The proof is in three steps:

Step 1. if equality (3.16) holds, then $\alpha_{n}=\gamma_{n} / \beta_{n}{ }^{3}$ where $\left\{\gamma_{n}\right\} \in l^{2}$.

Step 2. The property of $\left\{\alpha_{n}\right\}$ in Step 1 is used to prove that $\alpha_{n}=0$ for $n>N(N$ is the number in Theorem 3.2).

Step 3. We finish the proof by proving that $\alpha_{n}=0$ also for $n \leq N$.

Now we proceed to realize this program.

Step 1: Decaying properties of $\left\{\alpha_{n}\right\}$. In this step, we use the shorthand notation

$$
H^{1}=H^{1}\left([0, T] ; L^{2}(\Omega)\right) .
$$

We shall use the following lemma (proved in the Appendix).

Lemma 3.3. Let a sequence $\left\{\alpha_{n}\right\}$ be such that

$$
\Phi(x, t)=\sum_{n \in \mathbb{Z}^{\prime}} \alpha_{n} e^{i \beta_{n} t} \Psi_{n} \in H^{1}=H^{1}\left(0, T ; L^{2}(\Gamma)\right) .
$$

If $\left\{e^{i \beta_{n} t} \Psi_{n}\right\}$ is Riesz on a shorter interval $T-\epsilon$, then there exists $\left\{\delta_{n}\right\} \in l^{2}$ such that

$$
\alpha_{n}=\frac{\delta_{n}}{\beta_{n}}
$$


We single out from the series (3.16) those terms which correspond to indices in $\mathcal{J}$ (if any). Let

$$
F(t)=\sum_{n \in \mathcal{J}} \alpha_{n} S_{n}(t) \Psi_{n} \quad \text { if } \mathcal{J} \neq \emptyset, \quad F(t)=0 \text { otherwise. }
$$

This sum is finite, and for the indices in this sum, we have

$$
\begin{aligned}
S_{n}(t)=1 & +(\alpha+i) t \\
& +\int_{0}^{t}(t-r)\left\{e^{-\alpha r}\left(N^{\prime \prime}(r)+i N^{\prime}(r)\right)\right. \\
& \left.-\alpha^{2} \int_{0}^{r} N_{1}(r-s) S_{n}(s) \mathrm{d} s\right\} \mathrm{d} r .
\end{aligned}
$$

So, $S_{n}(t)$ does not depend on $n$ when $n \in \mathcal{J}$, and it is of class $H^{3}: F(t)$ is a fixed $H^{3}$ function (possibly zero).

When, in the next equalities, the index of the series is not explicitly indicated, we intend that it belongs to the set $\mathbb{Z}^{\prime} \backslash \mathcal{J}$.

Using (3.9) and (3.11), we rewrite (3.16) as

$$
\begin{aligned}
-\sum \alpha_{n} e^{i \beta_{n} t} \Psi_{n}= & F(t)+\alpha \sum \frac{\alpha_{n}}{\beta_{n}} \Psi_{n} \sin \beta_{n} t \\
& +\int_{0}^{t} N_{1}(t-s) \sum \alpha_{n}\left(e^{i \beta_{n} s}+\frac{\alpha}{\beta_{n}} \sin \beta_{n} s\right) \Psi_{n} \mathrm{~d} s \\
& -\int_{0}^{t} N_{1}(t-r) \sum \alpha_{n} \mu_{n} S_{n}(r) \Psi_{n} \mathrm{~d} r \\
& +N_{1}^{\prime}(0) \sum \int_{0}^{t} \frac{\alpha_{n} \mu_{n}}{\beta_{n}} \sin \beta_{n}(t-r) S_{n}(r) \Psi_{n} \mathrm{~d} r \\
& +\int_{0}^{t} N_{1}^{\prime \prime}(s) \sum \frac{\alpha_{n} \mu_{n}}{\beta_{n}} \int_{0}^{t-s} \sin \beta_{n}(t-s-r) S_{n}(r) \Psi_{n} \mathrm{~d} r \mathrm{~d} s \\
= & F(t)+f_{1}+f_{2}+f_{3}+f_{4}+f_{5} .
\end{aligned}
$$

We already know that $F \in H^{1}$. We prove $f_{i} \in H^{1}$ for every $i$.

The fact that $\left\{\Psi_{n} \sin \beta_{n} s\right\}$ and $\left\{\psi_{n} \cos \beta_{n} s\right\}$ are Riesz sequences in $L^{2}\left(G_{T}\right)$ (see Lemma 2.4) implies that $f_{1}$ and $f_{2}$ belong to $H^{1}$.

The series in $f_{3}$ converges in $L^{2}\left(G_{T}\right)$ and $N_{1}(t)$ is continuously differentiable, so that $f_{3} \in H^{1}$. 
We consider the function $f_{4}$, i.e., we consider the series

$$
\sum \frac{\alpha_{n} \mu_{n}}{\beta_{n}} \int_{0}^{t} \sin \beta_{n}(t-r) S_{n}(r) \Psi_{n} \mathrm{~d} r .
$$

Using (3.13) and $d \leq 3$, we see that this series converges in $L^{2}\left(G_{T}\right)$. A formal termwise differentiation gives:

$$
\sum \alpha_{n} \mu_{n} \int_{0}^{t} \cos \beta_{n}(t-r) S_{n}(r) \Psi_{n} \mathrm{~d} r .
$$

We replace $S_{n}(r)$ with its expression (3.13), and we get:

$$
\begin{array}{r}
\sum \alpha_{n} \mu_{n} \int_{0}^{t} \cos \beta_{n}(t-s) E_{n}(s) \Psi_{n} \mathrm{~d} s \\
-i N^{\prime}(0) \sum \frac{\alpha_{n}}{\beta_{n}} \int_{0}^{t} s \cos \beta_{n}(t-s) e^{i \beta_{n} s} \Psi_{n} \mathrm{~d} s \\
+\sum \frac{\alpha_{n}}{\beta_{n}^{2}} \int_{0}^{t} M_{n}(s) \cos \beta_{n}(t-s) \mathrm{d} s .
\end{array}
$$

The three series converge: the series $(\mathbf{A})$ and $(\mathbf{B})$ because the integrand are linear combinations of $\Psi_{n} \cos \beta_{n} t, \Psi_{n} \sin \beta_{n} t$ and $\Psi_{n} e^{i \beta_{n} t}$ : convergence follows from Lemma 2.4. Lemma 2.1 (i.e., $d \leq 3$ ) shows convergence of the series $(\mathbf{C})$. So we have $f_{4} \in H^{1}$ and its convolution with $N_{1}^{\prime \prime}$ (i.e., $f_{5}$ ) belongs to $H^{1}$ too.

In conclusion, using controllability of the telegraph equation in a shorter time,

$$
\sum \alpha_{n} e^{i \beta_{n} t} \Psi_{n} \in H^{1} \quad \text { hence } \alpha_{n}=\frac{\delta_{n}}{\beta_{n}}, \quad\left\{\delta_{n}\right\} \in l^{2} .
$$

We replace this expression of $\left\{\alpha_{n}\right\}$ in (3.17), and we equate the derivatives of both sides. We get

$$
\begin{aligned}
& -i \sum \delta_{n} e^{i \beta_{n} t} \Psi_{n}=F^{\prime}(t)+\alpha \sum \frac{\delta_{n}}{\beta_{n}} \Psi_{n} \cos \beta_{n} t \\
& +\int_{0}^{t} N_{1}^{\prime}(t-s) \sum \frac{\delta_{n}}{\beta_{n}}\left(e^{i \beta_{n} s}+\frac{\alpha}{\beta_{n}} \sin \beta_{n} s\right) \Psi_{n} \mathrm{~d} s \\
& -\int_{0}^{t} N_{1}^{\prime}(t-r) \sum \frac{\delta_{n} \mu_{n}}{\beta_{n}} S_{n}(r) \Psi_{n} \mathrm{~d} r
\end{aligned}
$$




$$
\begin{aligned}
& +N_{1}^{\prime}(0) \int_{0}^{t} \sum \frac{\delta_{n} \mu_{n}}{\beta_{n}} \cos \beta_{n}(t-r) S_{n}(r) \Psi_{n} \mathrm{~d} r \\
& +\int_{0}^{t} N_{1}^{\prime \prime}(s) \int_{0}^{t-s} \sum \frac{\delta_{n} \mu_{n}}{\beta_{n}} \cos \beta_{n}(t-s-r) S_{n}(r) \Psi_{n} \mathrm{~d} r \mathrm{~d} s .
\end{aligned}
$$

Arguments similar to the previous ones show that every series on the right hand side can be differentiated once more. The term in the second last line is the one that deserves a bit of attention. Its derivative is the sum of the two series

$$
N_{1}^{\prime}(0) \sum \frac{\delta_{n} \mu_{n}}{\beta_{n}} S_{n}(t) \Psi_{n},-N_{1}^{\prime}(0) \sum \delta_{n} \mu_{n} \int_{0}^{t} \sin \beta_{n}(t-r) S_{n}(r) \Psi_{n} \mathrm{~d} r .
$$

The first series converges thanks to the first statement in Theorem 3.2.

We insert (3.13) in the second series, and we get

$$
\sum \delta_{n} \mu_{n} \Psi_{n} \int_{0}^{t} \sin \beta_{n}(t-r)\left\{E_{n}(r)-i r N^{\prime}(0) \frac{1}{\beta_{n}} e^{i \beta_{n} r}+\frac{1}{{\beta_{n}}^{2}} M_{n}(r)\right\} \mathrm{d} r .
$$

Convergence of this series is seen as above. Hence, we get

$$
\delta_{n}=\frac{\widetilde{\gamma}_{n}}{\beta_{n}}, \quad \alpha_{n}=\frac{\widetilde{\gamma}_{n}}{{\beta_{n}{ }^{2}}^{2}}, \quad\left\{\widetilde{\gamma}_{n}\right\} \in l^{2} .
$$

Now we iterate this process: we replace $\delta_{n}$ with $\widetilde{\gamma}_{n} / \beta_{n}$, and we equate the derivatives. We get

$$
\widetilde{\gamma}_{n}=\frac{\gamma_{n}}{\beta_{n}}, \quad \text { i.e., } \alpha_{m}=\frac{\gamma_{n}}{\beta_{n}{ }^{3}}, \quad\left\{\gamma_{n}\right\} \in l^{2} .
$$

Details of the computations are in the Appendix.

In conclusion, we proved the existence of a sequence $\left\{\gamma_{n}\right\} \in l^{2}$ such that

$$
\alpha_{n}=\gamma_{n} \quad \text { if } \quad n \in \mathcal{J}, \quad \alpha_{n}=\frac{\gamma_{n}}{\beta_{n}{ }^{3}}, \quad \text { if } n \notin \mathcal{J},
$$

where $\alpha_{n}$ are the coefficients in the series (3.16).

Step 2: the sum in (3.16) is finite. We recall the definition of $S_{n}(t)$ in terms of $Z_{n}(t)$, and we rewrite (3.16) as

$$
\sum_{n \in \mathbb{Z}^{\prime}} \alpha_{n} \Psi_{n} Z_{n}(t)=\sum_{n \in \mathbb{Z}^{\prime}} \frac{\gamma_{n}}{\beta_{n}{ }^{3}} \Psi_{n} Z_{n}(t)=0 .
$$


The series (3.22) converges uniformly so that:

$$
\sum_{n \in \mathbb{Z}^{\prime}} \alpha_{n} \Psi_{n}=\sum_{n \in \mathbb{Z}^{\prime}} \frac{\gamma_{n}}{\beta_{n}^{3}} \Psi_{n}=0
$$

Here $\gamma_{n} / \beta_{n}{ }^{3}$ has to be replaced with $\gamma_{n}$ if $n \in \mathcal{J}$. We implicitly intend this substitution also in the next series.

The first statement in Theorem 3.2, the form of $K_{n}(t), d \leq 3$ and $\beta_{n}{ }^{2} \asymp \lambda_{n}^{2}$, show that the series (3.22) is termwise differentiable. Hence, we have:

$$
\sum \alpha_{n} \Psi_{n}\left\{-\lambda_{n}^{2} \int_{0}^{t} N(t-s) Z_{n}(s) \mathrm{d} s+K_{n}(t)\right\}=0 .
$$

We can distribute the series on the sum, and we get

$$
\int_{0}^{t} N(t-s) \sum_{n \in \mathbb{Z}^{\prime}} \frac{\gamma_{n} \lambda_{n}^{2}}{\beta_{n}{ }^{3}} Z_{n}(s) \Psi_{n} \mathrm{~d} s=\sum_{n \in \mathbb{Z}^{\prime}} \frac{\gamma_{n}}{\beta_{n}{ }^{3}} K_{n}(t) \Psi_{n} .
$$

Using (3.23) and $K_{n}(t)=N^{\prime}(t)+i \beta_{n} N(t)$, we get

$$
\sum \frac{\gamma_{n}}{\beta_{n}^{3}} K_{n}(t) \Psi_{n}=i N(t) \sum \frac{\gamma_{n}}{\beta_{n}^{2}} \Psi_{n}
$$

and so

$$
\int_{0}^{t} N(t-s) \sum_{n \in \mathbb{Z}^{\prime}} \frac{\gamma_{n} \lambda_{n}^{2}}{\beta_{n}{ }^{3}} Z_{n}(s) \Psi_{n} \mathrm{~d} s=i N(t) \sum_{n \in \mathbb{Z}^{\prime}} \frac{\gamma_{n}}{\beta_{n}{ }^{2}} \Psi_{n} .
$$

The property $N(0) \neq 0$ implies a further property of $\left\{\alpha_{n}\right\}$ :

$$
\sum_{n \in \mathbb{Z}^{\prime}} \frac{\gamma_{n}}{\beta_{n}^{2}} \Psi_{n}=\sum_{n \in \mathbb{Z}^{\prime}} \beta_{n} \alpha_{n} \Psi_{n}=0,
$$

and so the right hand side of (3.24) vanishes.

The property $N(0) \neq 0$ used in (3.24) gives:

$$
\sum_{n \in \mathbb{Z}^{\prime}} \alpha_{n} \lambda_{n}^{2} Z_{n}(t) \Psi_{n}=\sum_{n \in \mathbb{Z}^{\prime}} \frac{\gamma_{n} \lambda_{n}^{2}}{\beta_{n}^{3}} Z_{n}(t) \Psi_{n}=0 .
$$

We recall equality (3.22): $\sum_{n \in \mathbb{Z}^{\prime}} \alpha_{n} Z_{n}(t) \Psi_{n}=0$. We introduce the finite (possibly empty) set of indices

$$
\mathcal{O}=\left\{n: \lambda_{n}=0\right\}
$$


Note that if $n \in \mathcal{O}$ then $Z_{n}(t)=\widehat{Z}(t)$, the same for every $n$. We rewrite (3.26) and (3.22) as (the sum on the right side is zero if $\mathcal{O}=\emptyset$ ): (3.27)

$$
\sum_{n \notin \mathcal{O}} \alpha_{n} Z_{n}(t) \Psi_{n}=-\sum_{n \in \mathcal{O}} \alpha_{n} Z_{n}(t) \Psi_{n}, \quad \sum_{n \notin \mathcal{O}} \alpha_{n} \lambda_{n}^{2} Z_{n}(t) \Psi_{n}=0 .
$$

Let $k_{1} \notin \mathcal{O}$ be an index (of minimal absolute value) for which $\alpha_{k_{1}} \neq 0$. By combining the equalities in (3.27) we get

$$
\sum_{n \notin \mathcal{O}}\left(\alpha_{n}-\frac{\alpha_{n} \lambda_{n}^{2}}{\lambda_{k_{1}}^{2}}\right) Z_{n}(t) \Psi_{n}=-\sum_{n \in \mathcal{O}} \alpha_{n} Z_{n}(t) \Psi_{n} .
$$

Note that the right hand side is the same as in the first equality of (3.27).

Let

$$
\alpha_{n}^{(1)}=\left(1-\frac{\lambda_{n}^{2}}{\lambda_{k_{1}}^{2}}\right) \alpha_{n}
$$

and note that

$$
\left\{\alpha_{n}^{(1)}\right\} \in l^{2} ; \quad\left\{\begin{array}{l}
\alpha_{k_{1}}^{(1)}=0 \text { if } \lambda_{k}=\lambda_{k_{1}} \\
\text { if } \lambda_{k} \neq \lambda_{k_{1}} \text { then } \alpha_{k}^{(1)}=0 \Longleftrightarrow \alpha_{k}=0 .
\end{array}\right.
$$

So,

$$
\left\{\begin{array}{l}
\sum_{n \in \mathcal{O}} \alpha_{n} Z_{n}(t) \Psi_{n} \in X_{1}=\operatorname{cl} \operatorname{span}\left\{Z_{n}(t) \Psi_{n}, n \notin \mathcal{O}, \lambda_{n} \neq \lambda_{k_{1}}\right\} \\
\sum_{\substack{n \notin \mathcal{O} \\
\lambda_{n} \neq \lambda_{k_{1}}}} \alpha_{n}^{(1)} Z_{n}(t) \Psi_{n}=-\sum_{n \in \mathcal{O}} \alpha_{n} Z_{n}(t) \Psi_{n} .
\end{array}\right.
$$

Thanks to $\left\{\alpha_{n}^{(1)}\right\} \in l^{2}$, we can start a bootstrap argument and repeat this procedure: we find that $\left\{\lambda_{n}^{3} \alpha_{n}^{(1)}\right\} \in l^{2}$. We fix a second element $k_{2}$ (of minimal absolute value) such that $\alpha_{k_{2}}^{(1)} \neq 0$ and, as above, we get

$$
\left\{\begin{array}{r}
\sum_{n \in \mathcal{O}} \alpha_{n} Z_{n}(t) \Psi_{n} \in X_{2}=\operatorname{cl} \operatorname{span}\left\{Z_{n}(t) \Psi_{n}, n \notin \mathcal{O},\right. \\
\left.\lambda_{n} \notin\left\{\lambda_{k_{1}}, \lambda_{k_{2}}\right\}\right\} \\
\sum_{\substack{n \notin \mathcal{O} \\
\lambda_{n} \notin\left\{\lambda_{k_{1}}, \lambda_{k_{2}}\right\}}} \alpha_{n}^{(2)} Z_{n}(t) \Psi_{n}=\sum_{n \in \mathcal{O}} \alpha_{n} Z_{n}(t) \Psi_{n} .
\end{array}\right.
$$


The new sequence $\left\{\alpha_{n}^{(2)}\right\} \in l^{2}$ has the property that

$$
\left\{\begin{array}{l}
\alpha_{k}^{(2)}=0 \text { if } \lambda_{k} \in\left\{\lambda_{k_{1}}, \lambda_{k_{2}}\right\} \\
\text { if } \lambda_{n} \notin\left\{\lambda_{k_{1}}, \lambda_{k_{2}}\right\} \text { then } \alpha_{n}^{(2)}=0 \Longleftrightarrow \alpha_{n}=0 .
\end{array}\right.
$$

The argument can be repeated, and we find

$\sum_{n \in \mathcal{O}} \alpha_{n} Z_{n}(t) \Psi_{n} \in X_{R}=\operatorname{cl} \operatorname{span}\left\{Z_{n}(t) \Psi_{n}, n \notin \mathcal{O}, \lambda_{n} \notin\left\{\lambda_{k_{1}}, \lambda_{k_{2}}, \ldots \lambda_{k_{R}}\right\}\right\}$ for every $R$, i.e.

Lemma 3.4. We have:

$$
\sum_{n \in \mathcal{O}} \alpha_{n} Z_{n}(t) \Psi_{n} \in \bigcap_{R} X_{R}=\{0\}
$$

and, after at most $2 N$ iterations of the process, we find

$$
\sum_{|n|>N} \alpha_{n}^{(N)} Z_{n}(t) \Psi_{n}=0
$$

If $N$ is large enough, as specified in Theorem 3.2, we see that

$$
\alpha_{n}^{(N)}=0 \quad \text { when }|n|>N
$$

and the original equality (3.22) involves a finite sum. We rewrite it as

$$
\sum_{\substack{|n| \leq K \\ n \notin \mathcal{O}}} \alpha_{n} Z_{n}(t) \Psi_{n}=0
$$

Step 3: we have $\alpha_{n}=0$ for every $n$. We use the following lemma:

Lemma 3.5. The sequence $\left\{Z_{n}(t) \Psi_{n}(x)\right\}_{n \notin \mathcal{O}}$ is linearly independent.

The proof is similar to the proof of the corresponding result in $[\mathbf{3}, \mathbf{2 9}]$ and is omitted. This lemma and (3.30) imply $\alpha_{n}=0$ if $n \notin \mathcal{O}$.

In conclusion, equality (3.22) is in fact

$$
0=\sum_{n \in \mathcal{O}} \alpha_{n} Z_{n}(t) \Psi_{n}=\widetilde{Z}(t) \sum_{n \in \mathcal{O}} \alpha_{n} \Psi_{n}
$$


$\widetilde{Z}(t) \neq 0$ so that

$$
\sum_{n \in \mathcal{O}} \alpha_{n} \Psi_{n}=0
$$

Finally we prove:

Lemma 3.6. If $n \in \mathcal{O}$, then $\alpha_{n}=0$.

Proof. We introduce

$$
\Phi(x)=\sum_{n \in \mathcal{O}} \alpha_{n} \Phi_{n}(x)
$$

which is an eigenfunction of the operator $A$ whose eigenvalue is 0

$$
A \Phi(x)=0 .
$$

Note that if $\lambda_{n}=0$, then $\beta_{n}=i \alpha$ does not depend on $n$, and so

$$
\Psi_{n}= \begin{cases}\frac{\gamma_{a} \Phi_{n}}{\beta_{n}}=\frac{\gamma_{a} \Phi_{n}}{i \alpha} & \text { if } \alpha \neq 0 \\ \gamma_{a} \Phi_{n} & \text { if } \alpha=0 .\end{cases}
$$

So, in both cases, we get

$$
A \Phi=0, \quad \gamma_{a} \Phi=0 .
$$

By using statement (ii) in Theorem 2.6, we see that

$$
\Phi(x)=\sum \alpha_{n} \Phi_{n}(x)=0 .
$$

Condition $\alpha_{n}=0$ follows, since $\left\{\Phi_{n}\right\}$ is an orthonormal sequence.

4. Sharp control time. The results proved up to now show that the sharp control time of the viscoelastic system is not larger than that of the telegrapher's equation. Conversely controllability of (1.1) implies controllability of the telegrapher's equation (2.5). In fact, if equation (1.1) is controllable at time $T$ then the moment problem (3.4) is solvable (with continuity) and then the sequence $\left\{Z_{n}(t) \Psi_{n}\right\}$ is a Riesz sequence in $L^{2}\left(0, T ; L^{2}(\Gamma)\right)$. The first statement of Theorem 3.2 implies the existence of a number $N$ such that, for $|n|>N$, the sequence whose elements are described in Theorem 2.7 is Riesz in $L^{2}\left(0, T_{0} ; L^{2}(\Gamma)\right)$. 
This implies that the moment problem (2.21)-(2.22) for the telegrapher's equation is solvable for $\left\{\left(\xi_{n}, \eta_{n}\right)\right\} \in L$, where $L$ has finite codimension, see [10, page 323], i.e., the reachable set at time $T_{0}$ for the telegrapher's equation has finite codimension. We use Lemma 2.5 in order to prove that this is not true if the telegrapher's equation is not controllable.

Let $T>T_{0}$ be any time at which the telegrapher's equation (2.5) is controllable.

Let us denote $e_{n}$ the elements of the sequence described in Theorem 2.7. By adding elements of $\left(\operatorname{clspan}\left\{e_{n}\right\}\right)^{\perp}$, we complete the sequence $\left\{e_{n}\right\}$ to a Riesz basis of $L^{2}\left(0, T ; L^{2}(\Gamma)\right)$. We denote $k_{n}$ the added elements.

We consider the operator $\mathbb{J}_{0}: L^{2}\left(0, T_{0} ; L^{2}(\Gamma)\right) \mapsto l^{2}$ given by

$$
\mathbb{J}_{0} f=\left\{\left\langle f, e_{n}\right\rangle_{L^{2}\left(0, T_{0} ; L^{2}(\Gamma)\right)}\right\} \cup\left\{\left\langle f, k_{n}\right\rangle_{L^{2}\left(0, T_{0} ; L^{2}(\Gamma)\right)}\right\} .
$$

Lemma 2.5 shows that the codimension of its image is not finite, and so we also have

$$
\operatorname{dim} L^{\perp}=\operatorname{dim}\left\{\left\langle f, e_{n}\right\rangle_{L^{2}\left(0, T_{0} ; L^{2}(\Gamma)\right)}\right\}^{\perp}=+\infty .
$$

So, the index $N$ cannot exist and the viscoelastic system is not controllable at time $T_{0}$ if the telegrapher's equation is not controllable.

The previous negative result proves the second statement in Theorem 1.3, and it has a clear relation with the following fact, that the speed of propagation of waves in a viscoelastic body is equal to the speed of propagation in the corresponding (memoryless) elastic body, see $[6,7]$.

5. Appendix: proofs. Ancillary proofs are collected here.

In order to prove Lemma 2.4 we first note that the transformation

$$
\sum \alpha_{n} e^{i b_{n} t} k_{n} \longmapsto \sum \alpha_{n} e^{-i b_{n} T} e^{i b_{n} \tau} k_{n}: L^{2}(-T, T ; K) \longmapsto L^{2}(0,2 T ; K)
$$

is bounded and boundedly invertible since $\left\{\left|\mathcal{I} m b_{n}\right|\right\}$ is bounded. Hence, the assumption is that (2.9) holds for the sequence $\left\{e^{i b_{n} t} k_{n}\right\}_{n \in \mathbb{Z}^{\prime}}$ in $L^{2}(-T, T ; K)$. We use Euler formulas and we see that (2.9) holds for the cosine sequences $(2.11)$ in $L^{2}(0, T ; K)$ (the sine sequence is treated 
analogously). In fact:

$$
\begin{aligned}
\left\|\sum_{n>0} a_{n} k_{n} \cos b_{n} t\right\|_{L^{2}(0, T ; K)}^{2} & =\frac{1}{4}\left\|\sum_{n>0} a_{n} k_{n} e^{i b_{n} t}+\sum_{n>0} a_{n} k_{n} e^{-i b_{n} t}\right\|_{L^{2}(0, T ; K)}^{2} \\
& =\frac{1}{8}\left\|\sum_{n \in \mathbb{Z}^{\prime}} a_{n} k_{n} e^{i b_{n} t}\right\|_{L^{2}(-T, T ; K)}^{2} .
\end{aligned}
$$

In the last equality, we put $a_{n}=a_{-n}$, and we used $-b_{n}=b_{n}, k_{-n}=k_{n}$.

Inequalities (2.9) hold by assumption for the right side and so they hold also for the left side.

This proof has been adapted from [11], where it is proved that the opposite implication is false.

Now we prove Lemma 2.5. We know that

$$
\left(\mathcal{I} m \mathbb{J}_{0}\right)^{\perp}=\operatorname{ker} \mathbb{J}_{0}^{*}=\left\{\left\{c_{n}\right\} \in l^{2}: \sum c_{n} e_{n}=0 \quad \text { in } L^{2}\left(0, T_{0} ; K\right)\right\} .
$$

Every sequence $\left\{c_{n}\right\} \in l^{2}$ such that $\sum c_{n} e_{n}=0$ in $L^{2}\left(0, T_{0} ; K\right)$ while $\sum c_{n} e_{n} \neq 0$ in $L^{2}\left(T_{0}, T ; K\right)$ belongs to ker $\mathbb{J}_{0}^{*}$, and conversely. So, $\operatorname{dim}$ ker $\mathbb{J}_{0}^{*}=+\infty$.

Real and complex solutions of two moment problems. We use $(\cdot, \cdot)$ to denote the integral of a product in $L^{2}\left(G_{T}\right)$ (so that $(f, g)$ is linear in both the entries) and we use $l^{2}(\mathbb{N})$ and $l^{2}\left(\mathbb{Z}^{\prime}\right)$ to denote the space of the complex valued $l^{2}$ sequences, with indices in $\mathbb{N}$ or in $\mathbb{Z}^{\prime}$.

Let $e_{n}=\left\{\zeta_{n}+i \hat{\zeta}_{n}\right\}$ be a sequence in $L^{2}\left(G_{T}\right)$ such that $e_{n}=-\bar{e}_{-n}$ so that

$$
\zeta_{-n}=-\zeta_{n}, \quad \widehat{\zeta}_{-n}=\widehat{\zeta}_{n} .
$$

We consider the problems

$$
\begin{array}{lll}
\left(e_{n}, f\right)=c_{n}, & \left\{c_{n}\right\}=\left\{\eta_{n}+i \xi_{n}\right\} \in l^{2}(\mathbb{N}) \\
\left(e_{n}, g\right)=d_{n}=r_{n}+i s_{n}, & & \left\{d_{n}\right\} \in l^{2}\left(\mathbb{Z}^{\prime}\right) .
\end{array}
$$

We prove that if the problem (5.1) has a real valued solution $f \in$ $L^{2}\left(G_{T}\right)$ which depends continuously on $\left\{c_{n}\right\} \in L^{2}(\mathbb{N})$, then moment problem (5.2) has a complex valued solution $g \in L^{2}\left(G_{T}\right)$, which depends continuously on $\left\{d_{n}\right\} \in l^{2}\left(\mathbb{Z}^{\prime}\right)$, and conversely. Let $g=$ $h+i k$. We separate real and imaginary parts and we see that moment 
problem (5.2) can be reformulated as

$$
\begin{array}{cl}
\left(\xi_{n}, h\right)-\left(\widehat{\zeta}_{n}, k\right)=r_{n}, & \left(\zeta_{n}, k\right)+\left(\widehat{\zeta}_{n}, h\right)=s_{n} \\
\left(\zeta_{n}, h\right)+\left(\widehat{\zeta}_{n}, k\right)=-r_{-n}, & \left(\zeta_{n}, k\right)-\left(\widehat{\zeta}_{n}, h\right)=-s_{-n} .
\end{array}
$$

This is equivalent to the pair of problems (both with arbitrary complex valued $l^{2}$ sequences on the right hand side)

$$
\begin{aligned}
\left(\zeta_{n}+i \widehat{\zeta}_{n}, h\right) & =\frac{1}{2}\left\{\left[r_{n}-r_{-n}\right]+i\left[s_{n}+s_{-n}\right]\right\} \quad n \in \mathbb{N}, h \text { real valued. } \\
\left(\zeta_{n}+i \widehat{\zeta}_{n}, k\right) & =\frac{1}{2}\left\{\left[s_{n}-s_{-n}\right]-i\left[r_{n}+r_{-n}\right]\right\} n \in \mathbb{N}, k \text { real valued. }
\end{aligned}
$$

So, the solution of (5.2) is the same as the solution of two copies of problem (5.1). This ends the proof.

The proof of Lemma 3.3. We first note that Theorem 3.2 implies $\left\{\alpha_{n}\right\} \in l^{2}$ and that, in order to prove the formula for $\left\{\alpha_{n}\right\}$, it is sufficient that we prove that it holds for $|n|$ sufficiently large. So, we consider the new function

$$
C(x, t)=\sum_{|n| \geq N} \alpha_{n} e^{i \beta_{n} t} \Psi_{n} \in W^{1,2}\left(0, T+\widehat{h} ; L^{2}(\Gamma)\right),
$$

where $N$ is the number specified in Theorem 3.2. It is known that $C_{t}(x, t) \in L^{2}\left(0, T ; L^{2}(\Gamma)\right)$ is the limit of the incremental quotient:

$$
C_{t}(x, t)=\lim _{h \rightarrow 0} \frac{C(x, t+h)-C(x, t)}{h}=\lim _{h \rightarrow 0} \sum_{|n|>N} \alpha_{n} \frac{e^{i \beta_{n} h}-1}{h} e^{i \beta_{n} t} \Psi_{n} .
$$

Thanks to the choice of $N$, there exists $m_{0}>0$ such that

$$
\begin{aligned}
m_{0} \sum_{|n|>N}\left|\alpha_{n} \beta_{n} \frac{e^{i \beta_{n} h}-1}{\beta_{n} h}\right|^{2} & \leq\left\|\frac{C(x, t+h)-C(x, t)}{h}\right\|_{L^{2}\left(0, T ; L^{2}(\Gamma)\right)}^{2} \\
& \leq 2\left\|C^{\prime}\right\|_{L^{2}\left(0, T ; L^{2}(\Gamma)\right)}^{2} .
\end{aligned}
$$

The last equality holds for $h$ "small," $|h|<h_{0}$. We consider $0<h<h_{0}$.

Let $s$ be real. There exists $s_{0}>0$ such that:

$$
\left|\frac{e^{i s}-1}{s}\right|^{2}=\left(\frac{\cos s-1}{s}\right)^{2}+\left(\frac{\sin s}{s}\right)^{2}>\frac{1}{2} \quad \text { for } 0<s<s_{0} .
$$


Then we have, for every $h \in\left(0, h_{0}\right)$,

$$
\frac{1}{2} \sum_{\substack{|n|>N \\ \beta_{n}<s_{0} / h}}\left|\alpha_{n} \beta_{n}\right|^{2} \leq \sum_{|n|>N}\left|\alpha_{n} \beta_{n} \frac{e^{i \beta_{n} h}-1}{\beta_{n} h}\right|^{2} \leq \frac{2}{m_{0}}\left\|C^{\prime}\right\|_{L^{2}\left(0, T ; L^{2}(\Gamma)\right)}^{2} .
$$

The limit for $h \rightarrow 0^{+}$gives the result.

End of the proof of formula (3.21). We insert $\delta_{n}=\widetilde{\gamma}_{n} / \beta_{n}$ in (3.20), and we equate the derivatives of both sides. The right hand side is the sum of $F^{\prime \prime}(t) \Psi_{n}$ which can be differentiated, and of the following functions $\mathbf{S}_{\mathbf{1}}-\mathbf{S}_{\mathbf{5}}$, where

$$
\begin{aligned}
& \mathbf{S}_{\mathbf{1}}=-\alpha \sum \frac{\tilde{\gamma}_{n}}{\beta_{n}} \Psi_{n} \sin \beta_{n} t \\
& \mathbf{S}_{\mathbf{2}}=N_{1}^{\prime}(0) \sum \frac{\widetilde{\gamma}_{n}}{\beta_{n}{ }^{2}} \Psi_{n}\left(e^{i \beta_{n} t}+\frac{\alpha}{\beta_{n}} \sin \beta_{n} t\right) \\
& \mathbf{S}_{\mathbf{3}}=\int_{0}^{t} N_{1}^{\prime \prime}(t-s) \sum \frac{\widetilde{\gamma}_{n}}{\beta_{n}{ }^{2}} \Psi_{n}\left(e^{i \beta_{n} s}+\frac{\alpha}{\beta_{n}} \sin \beta_{n} s\right) \mathrm{d} s .
\end{aligned}
$$

These functions can be differentiated since $\left\{\Psi_{n} \sin \beta_{n} t\right\}$ and $\left\{\Psi_{n} \cos \beta_{n} t\right\}$ are Riesz sequences in $L^{2}\left(G_{T}\right)$.

The remaining functions are

$$
\begin{aligned}
& \mathbf{S}_{\mathbf{4}}=-N_{1}^{\prime}(0) \sum \int_{0}^{t} \frac{\widetilde{\gamma}_{n} \mu_{n}}{\beta_{n}} \sin \beta_{n}(t-r) \Psi_{n} S_{n}(r) \mathrm{d} r \\
& \mathbf{S}_{\mathbf{5}}=-\int_{0}^{t} N_{1}^{\prime \prime}(s)\left[\sum \int_{0}^{t-s} \frac{\tilde{\gamma}_{n} \mu_{n}}{\beta_{n}} \sin \beta_{n}(t-s-r) \Psi_{n} S_{n}(r) \mathrm{d} r\right] \mathrm{d} s .
\end{aligned}
$$

The series in $\mathbf{S}_{\mathbf{4}}$ is $L^{2}\left(G_{T}\right)$ convergent. Termwise differentiation gives $\frac{\mathrm{d}}{\mathrm{d} t} \int_{0}^{t} \sum \frac{\widetilde{\gamma}_{n} \mu_{n}}{\beta_{n}} \sin \beta_{n}(t-r) S_{n}(r) \mathrm{d} r=\int_{0}^{t} \sum \widetilde{\gamma}_{n} \mu_{n} \cos \beta_{n}(t-r) S_{n}(r) \mathrm{d} r$.

This series is $L^{2}\left(G_{T}\right)$-convergent and so the series in $\mathbf{S}_{\mathbf{4}}$ belongs to $H^{1}$ (compare with the series (3.19)). This implies differentiability of $\mathbf{S}_{\mathbf{5}}$.

Acknowledgments. The author thanks the referees for their useful suggestions, which improved the readability of the paper. 


\section{REFERENCES}

1. S. Avdonin and B.P. Belinskiy, On controllability of an homogeneous string with memory, J. Math. Anal. Appl. 398 (2013), 254-269.

2. S.A. Avdonin and S.A. Ivanov, Families of exponentials. The method of moments in controllability problems for distributed parameter systems, Cambridge University Press, New York, 1995.

3. S. Avdonin and L. Pandolfi, Simultaneous temperature and flux controllability for heat equations with memory, Quart. Appl. Math. 71 (2013), 339-368.

4. _ Temperature and heat flux dependence/independence for heat equations with memory, in Time delay systems - methods, applications and new trend, R. Sipahi, et al., eds., Lect. Notes Contr. Inf. Sci. 423, Springer-Verlag, New York, 2012.

5. V. Barbu and M. Iannelli, Controllability of the heat equation with memory, Diff. Int. Eq. 13 (2000), 1393-1412.

6. G.M.C. Fisher and M.E. Gurtin, Wave propagation in the linear theory of viscoelasticity, Quart. Appl. Math. 23 (1965), 257-263.

7. I. Herrera and M.E. Gurtin, A correspondence principle for viscoelastic wave propagation, Quart. Appl. Math. 22 (1965), 360-364.

8. X. Fu, J. Yong and X. Zhang, Controllability and observability of the heat equation with hyperbolic memory kernel, J. Diff. Equat. 247 (2009), 2395-2439.

9. D. Fujiwara, Concrete characterization of the domains of fractional powers of some elliptic differential operators of the second order, Proc. Japan Acad. 43 (1967), 82-86.

10. I.C. Gohberg and M.G. Krejn, Opèrateurs Linèairs non Auto-adjoints dans un Espace Hilbertien, Dunod, Paris, 1971.

11. G.M. Gubreeve and M.G. Volkova, One remark about the unconditional exponential bases and cosine bases, connected with them, Meth. Funct. Anal. Topol. 14 (2008), 330-333.

12. M.E. Gurtin and A.G. Pipkin, A general theory of heat conduction with finite wave speed, Arch. Rat. Mech. Anal. 31 (1968), 113-126.

13. A. Hassel and T. Tao, Erratum for "Upper and lower bounds for normal derivatives of Dirichlet eigenfunctions," Math. Res. Lett. 17 (2010), 793-794.

14. J.U. Kim, Control of a second-order integro-differential equation, SIAM J. Contr. Optim. 31 (1993), 101-110.

15. I. Lasiecka, Controllability of a viscoelastic Kirchhoff plate, in Control and estimation of distributed parameter systems, Inter. Num. Math. 91 (1988), Birkhäuser, Basel, 237-247.

16. G. Leugering, Exact controllability in viscoelasticity of fading memory type, Appl. Anal. 18 (1984), 221-243.

17. $\quad$ On boundary controllability of viscoelastic systems, in Control of partial differential equations, Lect. Notes Contr. Inf. Sci. 114, Springer, Berlin, 1989. 
18. J.L. Lions, Contrôlabilitè exacte, perturbations et stabilization de systémes distribuès, 1, Masson, Paris, 1988.

19. P. Loreti, L. Pandolfi and D. Sforza, Boundary controllability and observability of a viscoelastic string, SIAM J. Contr. Optim. 50 (2012), 820-844.

20. J.U. Kim, Control of a second-order integro-differential equation, SIAM J. Contr. Optim. 31 (1993), 101-110.

21. V. Komornik, Exact controllability and stabilization. The multiplier method, John Wiley \& Sons, Ltd., Chichester, 1994.

22. S. Micu and E. Zuazua, An introduction to the controllability of partial differential equations, in Quelques questions de théorie du contròle, T. Sari, ed., Coll. Trav. Cours Hermann, 2004.

23. V.P. Mikhailov, Partial differential equations, Mir, Moscou, 1978.

24. L. Pandolfi, The controllability of the Gurtin-Pipkin equation: A cosine operator approach, Appl. Math. Optim. 52 (2005), 143-165. (see a correction in Appl. Math. Optim. 64 (2011) 467-468).

25. , Riesz systems and the controllability of heat equations with memory, Int. Eq. Oper. Theor. 64 (2009), 429-453.

26. _ Riesz systems and moment method in the study of heat equations with memory in one space dimension, Discr. Cont. Dynam. Syst. 14 (2010), 14871510 .

27. , Riesz systems and an identification problem for heat equations with memory, Discr. Cont. Dynam. Syst. 4 (2011), 745-759.

28. , Boundary controllability and source reconstruction in a viscoelastic string under external traction, J. Math. Anal. Appl. 407 (2013), 464-479.

29. , Traction, deformation and velocity of deformation in a viscoelastic string, Evol. Equat. Contr. Theor. 2 (2013), 471-493.

30. _ Distributed systems with persistent memory: Control and moment methods, Springer, New York, 2014.

31. A.C. Pipkin, Lectures on viscoelasticity theory, Springer-Verlag, New York, 1972 .

32. J.A. Soriano, Exact controllability of the generalized telegraph equation, Rev. Mat. Univ. Comp. Madrid 8 (1995), 459-493.

33. R.M. Young, An introduction to nonharmonic Fourier series, Academic Press, San Diego, CA, 2001.

Dipartimento di Scienze Matematiche "Giuseppe Luigi Lagrange," Politecnico di Torino, Corso Duca degli Abruzzi 24, 10129 Torino, Italy

Email address: luciano.pandolfi@polito.it 